\title{
Cosmic background dipole measurements with the Planck-High Frequency Instrument
}

\author{
M. Piat ${ }^{1}$, G. Lagache ${ }^{1}$, J. P. Bernard ${ }^{1}$, M. Giard ${ }^{2}$, and J. L. Puget ${ }^{1}$ \\ 1 Institut d'Astrophysique Spatiale (IAS), Université Paris Sud, Bât. 121, 91405 Orsay Cedex, France \\ 2 Centre d'Etude Spatiale des Rayonnements (CESR), 9 avenue du Colonel Roche, BP 4346, 31028 Toulouse Cedex 4, France
}

Received 20 October 2001 / Accepted 28 June 2002

\begin{abstract}
This paper discusses the Cosmic Background (CB) dipole observations in the framework of the Planck mission. Dipole observations can be used in three ways: (i) It gives a measurement of the peculiar velocity of our Galaxy which is an important observation for large-scale structure formation models. (ii) Measuring the dipole can give information on the monopole that can be in some cases hard or impossible to measure due to large foreground contamination or instrumental design. (iii) The dipole can be an ideal absolute calibrator, easily detectable in cosmological experiments. Here, we study the accuracy of the Planck-HFI calibration using the Cosmic Microwave Background (CMB) dipole measured by COBE as well as the Earth orbital motion dipole. We show that for HFI we can reach a relative calibration between rings of about $1 \%$ and an absolute calibration better than $0.4 \%$ for the CMB channels. In the end, the absolute calibration will be limited by the uncertainties on the CMB temperature. We also show that Planck will be able to measure the CMB dipole direction at better than 1.7 arcmin and improve the accuracy of the amplitude. Second, we investigate the detection of the Cosmic Far-Infrared Background (FIRB) dipole. Measuring this dipole could give a new and independent determination of the FIRB for which a direct determination is quite difficult. We show that such a detection would require Galactic dust emission removal to better than $1 \%$, which would be very hard to achieve.
\end{abstract}

Key words. cosmology: observations - cosmology: cosmic microwave background - space vehicules: instruments

\section{Introduction: The Cosmic Background and its dipole signal}

The Cosmic Background (CB) is the extragalactic part of the diffuse electromagnetic emission at all wavelengths. If the universe obeys the cosmological principle and is homogeneous and isotropic, this background is expected to be nearly isotropic in the rest frame where the matter in a large volume around the observer has no bulk velocity other than the Hubble expansion. The measurement of the intensity and Spectral Energy Distribution (SED) of this isotropic background is a difficult observational challenge. It requires a separation of this extragalactic component from all other diffuse foregrounds (interplanetary or interstellar emission). Although the CB was detected quite early in the radio (radio-galaxies), $\mathrm{X}$ rays and gamma rays as it dominates over the foregrounds, these components account for only $0.027 \%$ of the electromagnetic content of the present universe. The $\mathrm{CB}$ is dominated by the Cosmic Microwave Background (CMB) which accounts for $93 \%$ of the electromagnetic content of the present universe and is a truly diffuse component coming from the pre-galactic era of the universe. The second component in energy content is the radiation

Send offprint requests to: M. Piat, e-mail: michel.piat@ias.u-psud.fr from galaxies integrated over all redshifts in the ultravioletoptical-near-infrared (stellar emission) and in the thermal and far infrared (re-emission of stellar radiation absorbed by interstellar dust). This component has been detected only recently, first in the far infrared in the COBE-FIRAS and COBE-DIRBE data (Puget et al. 1996; Fixsen et al. 1998; Hauser et al. 1998; Lagache et al. 2000) then in the optical with HST (Bernstein et al. 2001; Pozzetti et al. 1998) and near infrared (Gorjian et al. 2000; Dwek \& Arendt 1998; Cambrésy et al. 2000; Wright 2001). These measurements have been complemented by very high-energy gamma ray absorption by this CB (see for example Stanev \& Franceschini 1998; Renault et al. 2001) but are still affected by rather large uncertainties especially in the optical and mid infrared. Figure 1 shows the spectrum of the CB from radio to gamma rays (data from Gispert et al. 2000 for the part [30 nm; $1 \mathrm{~mm}$ ] and from Halpern \& Scott 1999 for the radio part).

The CB should display a dipole anisotropy associated with the peculiar motion of the observer with respect to the local cosmological standard of rest. This peculiar motion was first detected for the CMB by Kogut et al. (1993). It is composed of several terms: the peculiar motion of the Sun (sum of the Galaxy peculiar velocity and the velocity of the Sun due to the rotation of our Galaxy), the Earth velocity along its orbit 
around the Sun and the specific motion of the observer with respect to the Earth (orbital motion of the satellite for example). The main term is the first one with a velocity of about $369 \mathrm{~km} \mathrm{~s}^{-1}$ in the direction $(l, b) \simeq\left(264.3^{\circ}, 48.0^{\circ}\right)$ (Lineweaver et al. 1996). The second contributor of the observer peculiar motion is the Earth orbital motion, which induces a sinusoidal term with an amplitude of about $30 \mathrm{~km} \mathrm{~s}^{-1}$. It is thus about $8 \%$ of the first one but is perfectly known in amplitude and direction and changes periodically with time during the year. The COBE-DMR experiment has measured these terms accurately. Fixsen et al. (1996) has shown that the CMB spectrum absolutely measured with the COBE-FIRAS experiment with high accuracy could also be measured using its dipole anisotropy. The two spectra obtained were compatible within error bars. The peculiar motion of the galaxy has also been measured with respect to distant galaxies as an anisotropy in the Hubble constant (Lauer \& Postman 1994; Blake \& Wall 2002). Although the accuracy is not very high, the value obtained is compatible with that measured by COBE (Scaramella et al. 1991; Kogut et al. 1993). These measurements confirm that the rest frame defined as the one in which the CMB is isotropic coincides with the rest frame defined by the galaxies on large scales.

Motion with velocity $\boldsymbol{\beta}=\boldsymbol{v} / \boldsymbol{c}$ through an isotropic radiation field of intensity $I(v)$ yields an observed intensity given by:

$I_{\mathrm{obs}}(\nu, \theta)=\frac{I[v(1-\beta \cos \theta)]}{(1-\beta \cos \theta)^{3}}$

where $v$ is the frequency and $\theta$ is the angle between $\beta$ and the direction of observation, these two parameters beeing measured in the observer's frame. The spectral intensity of the dipole amplitude is therefore given by:

$$
\begin{aligned}
I_{\mathrm{dip}}(v) & =I_{\mathrm{obs}}\left(v, \theta=0^{\circ}\right)-I_{\mathrm{obs}}\left(v, \theta=90^{\circ}\right) \\
& \simeq \beta \cos \theta\left(3 I(v)-v \frac{\mathrm{d} I}{\mathrm{~d} v}(v)\right) \text { for } \beta \ll 1 .
\end{aligned}
$$

The spectral intensity of the CB dipole amplitude is shown in Fig. 1 and its relative level in Fig. 2.

The dipole anisotropy of the CB has a two-fold interest for future $\mathrm{CB}$ projects:

- at frequencies where the separation of the CB from foregrounds is difficult (from submillimeter to ultraviolet), an accurate measurement of the dipole anisotropy will give the SED of the CB independently of the interstellar and interplanetary foregrounds, which do not show a dipole anisotropy;

- a very accurate measurement of the CMB dipole anisotropy over a one year period should allow to use the dipole anisotropy associated with the orbital motion of the Earth around the Sun as the best photometric calibrator for extended sources in the microwave region of the spectrum. It should therefore improve the determination of the peculiar motion of the solar system. This is possible due to the Planckian nature of the CMB and the accuracy of its temperature measured with the FIRAS spectrometer. The CMB today provides an extended photometric standard with an accuracy better than $2 \times 10^{-3}$ in thermodynamic temperature (Fixsen et al. 1996).

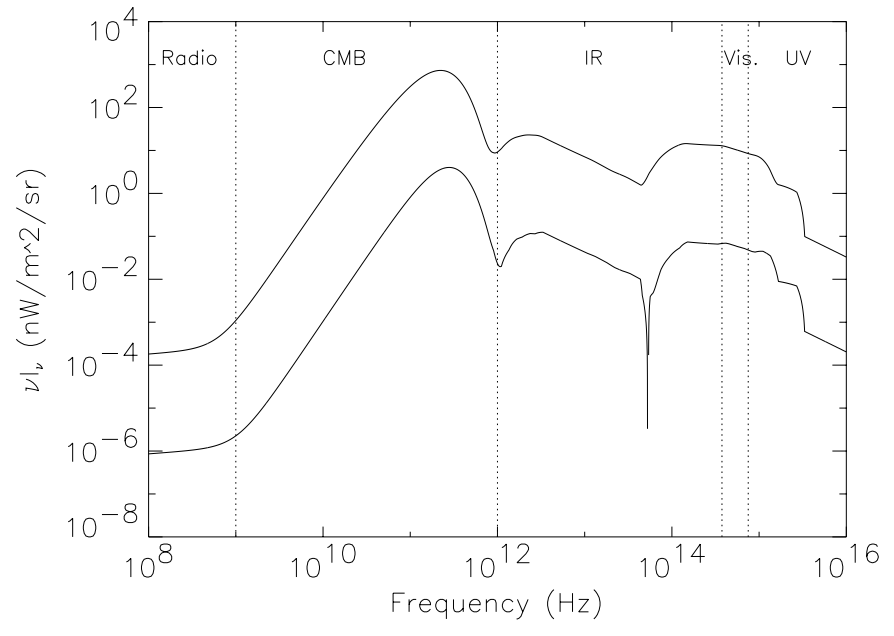

Fig. 1. Spectrum of the CB (top) and its dipole amplitude (bottom) from radio to ultra-violet.

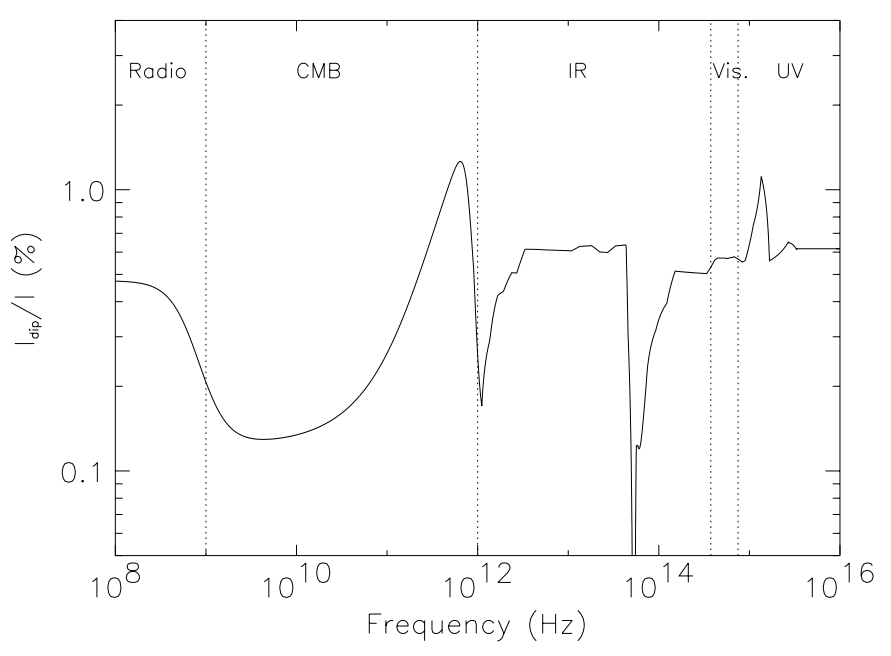

Fig. 2. Amplitude of the $\mathrm{CB}$ dipole relative to the $\mathrm{CB}$ intensity expressed in \% from radio to ultra-violet.

This paper explores these two questions in the context of the Planck mission and specifically its High Frequency Instrument (Planck-HFI, Lamarre 1998). It is divided in 2 parts. The first one deals with the calibration of Planck-HFI using the CMB dipoles. In Sect. 2, we present the calibration philosophy of Planck, while Sect. 3 concerns more specifically the calibration accuracy. The second part, in Sect. 4, is a study of the detectability of the Far InfraRed Background (FIRB) dipole.

\section{Planck-HFI calibration philosophy}

Different sources will be used to monitor the response inflight or to get the photometric calibration of the Planck-HFI channels:

- extended sources: the CMB dipole, the Earth orbital motion dipole, the Galactic disc and eventually maps from Herschel-SPIRE;

- point sources: planets, infrared galaxies and asteroids. 
It should be noted that the spectral intensity of the CMB monopole measured with high precision by COBE-FIRAS cannot be used as a source of calibration for Planck-HFI. Since bolometer detectors have a non-linear output response with respect to the input power, a small and known signal modulation is needed to determine the photometric calibration. HFI has been designed to map small CMB fluctuations and is therefore not sensible to the monopole term.

A general principle throughout the calibration for all channels is that relative calibrations will be established as precisely as possible between individual scans before establishing an absolute calibration for all data.

As will be seen in the next section, for channels dominated by the CMB (100 GHz to $217 \mathrm{GHz}$ ), the dipole signal from the $\mathrm{CMB}$ can be used to measure the relative response variations to the first order on all useful timescales since it is an extended source available over the whole mission. The amplitude and direction of the cosmic dipole has been measured by COBE. It will be remeasured independently by Planck using the dipole from the Earth orbital motion as the primary calibrator of the instrument absolute response.

For higher frequency channels for which signal is dominated by galactic dust emission, the calibration will be done by comparison with COBE-FIRAS map. The absolute calibration will also be done on the whole sky. The method to correct for relative reponse variations on different time scales is out of the scope of this paper. A short summary of the technique used is given below.

Relative calibration on a timescale of a week or more will be obtained with COBE-FIRAS data due to their large angular resolution (about $7^{\circ}$ ). On shorter timescales, a model of the detection chain could be used. The response variation of a bolometer depends on a few fixed (or very slowly varying) parameters such as the detector thermal conductance and the bias current. It also depends on changing parameters, mainly the temperature of the heat sink of the bolometer and the incident power background. This last quantity is directly related to the temperature of the various stages of the optics $(1.6 \mathrm{~K}, 4 \mathrm{~K}$ and the telescope) and to the flux from the sky which is known to first order before the mission or after a first iteration of the data reduction process. Since temperature of the optics is monitored during the mission, it will be possible to correlate the response measured as a function of time with the temperature of the relevant elements and adjust very precisely the parameters of the model describing the bolometer chain. This can be done very accurately using the slow variations of the different temperatures. This model with the temperature measurements (or any other monitored parameter found to affect the response) can be used to interpolate, if needed, the short time scale response variations in high frequency channels.

Relative variations of the response will thus be established on many time scales. A preliminary absolute calibration will be built after typically a month period and will be improved until the final full data reduction for a given detector is completed.

\section{Using the $\mathrm{CB}$ dipole as a calibration signal for HFI}

The CMB dipole is a strong known source (using COBE data initially or its properties remeasured by Planck at the end of the mission) which can be used to monitor the response variations over various time scales for all channels where its signal either dominates or is clearly detectable. It is thus important to evaluate the achievable accuracies for both the final absolute calibration and the measurements of the relative response variations.

The CMB shows anisotropies on all scales tracing the small inhomogeneities in the pre-galactic era that lead to the formation of structures in the universe. These fluctuations are observed on a surface of last scattering as seen from the Earth. They contain high spatial frequency terms which are the main objective of the Planck mission and low frequency components which do not carry much cosmological information as they are much more variable with the observer position (cosmic variance). The dipole term of this cosmological component is indistinguishable from the one associated with the peculiar motion of the solar system and is therefore a fundamental limit for the accuracy in measuring this motion. The CMB cosmological anisotropies are thus defined as having no dipole component $(\ell=1$ term of the usual decomposition in spherical harmonics of the sky). The contribution of higher $\ell$ to the dipole measurement is zero when obtained on the whole sky (by definition) but is not zero when the dipole is fitted on a fraction of the sky. In this section, we first present the Planck-HFI simulations we used, and explore the relative calibration on rings as well as the global absolute calibration accuracy.

\subsection{Simulations}

The Planck satellite will orbit around the lagrangian L2 point and will therefore follow the Earth orbital motion around the Sun. Its scanning strategy consists of rotating the satellite at $1 \mathrm{rpm}$ around its spin axis which will follow the antisolar direction. The beam axis, located $85^{\circ}$ away from the spin axis, scans the sky along large circles. We define a "circle" as beeing one instantaneous Planck observation of $1 \mathrm{~min}$. The spin axis is repointed about every hour so that each circle is scanned about 60 times. The beam will therefore cover the whole sky in six months. These 60 circles are averaged into what we call one "ring". In our simulation, the spin axis follows a sinusoidal trajectory along the ecliptic plane with 6 oscillations per year and with a full amplitude of $\pm 10^{\circ}$ so that the polar caps are not left unobserved.

For simplicity and to spare computer time and space, only 84 rings distributed uniformly on the sky over a one year mission were simulated. The simulated sky is the sum of 5 contributions:

1. CMB cosmological anisotropies, obtained from a standard Cold Dark Matter model: $\Omega_{\mathrm{tot}}=1, \Omega_{\mathrm{b}}=0.05, \Omega_{\Lambda}=0$ and $H_{0}=50 \mathrm{~km} \mathrm{~s}^{-1} / \mathrm{Mpc}$. The $C_{\ell}$ power spectrum is computed with CMBFAST (Seljak et al. 1996) and the map is generated in a Healpix-type all-sky pixelisation (Górski et al. 1996) with a pixel size of 3.5 arcmin; 
2. the solar system Peculiar Motion dipole (PM dipole) assuming an amplitude of $3.36 \mathrm{mK}$ in the direction $(l, b)=$ $\left(264.31^{\circ}, 48.05^{\circ}\right)($ Lineweaver et al. 1996);

3. the Earth Orbital Motion dipole (OM dipole) assuming an amplitude of about $336 \mu \mathrm{K}$ with its maximum at zero ecliptic latitude in the direction of the OM;

4. galactic dust, scaled from IRAS $100 \mu$ m map using a $17.5 \mathrm{~K}$ blackbody modified by a $v^{2}$ emissivity law (Boulanger et al. 1996), assuming that $90 \%$ is removed;

5. one realisation of the noise with levels given in Table 1. These numbers were deduced from the sensitivities of the Planck-HFI channels as derived by Lamarre et al. (2001). We include a $1 / f^{2}$ contribution to the noise power spectrum with a knee frequency of $10 \mathrm{mHz}$. In order to define the noise level for rings, the instantaneous sensitivity on a circle has been divided by $\sqrt{60}$, assuming that all circles are coadded into a single ring and that the noise is not correlated from one circle to the other. This is not strictly the case given the frequency aliasing produced by the co-addition (Janssen et al. 1996; Delabrouille 1998b). However, it is a rather good approximation (see Fig. 3 from Giard et al. 1999). Moreover, the removal of slow drifts on circles has to be considered together with the process of removing systematic effects using redundancies and is therefore out of the scope of this paper. For simplicity, slow drifts at frequencies lower than the spin frequency $f_{\text {spin }}=1 / 60 \mathrm{~Hz}$ are assumed to be removed using a destriping algorithm (e.g. Delabrouille 1998c).

The simulation did not include the Inter-Planetary Dust (IPD) emission. This contribution has been estimated from COBEFIRAS maps which are extrapolations from the model of Kelsall et al. (1998) based on COBE-DIRBE observations. A power law emissivity has been taken to extrapolate at the Planck-HFI wavelengths. Assuming that $80 \%$ of this contribution can be removed by proper modelling, the CMB dipole appeared to be at least an order of magnitude higher than the IPD emission dipole term (which decreases with wavelength) for all frequencies covered by Planck-HFI. This analysis showed that IPD emission is negligible for the present study.

\subsection{Ring analysis}

For each ring, a linear regression is done between the simulated signal and the expected dipole signal which is the sum of the PM dipole and the OM dipole as shown in Fig. 3. The slope of this linear regression gives the absolute calibration factor with its uncertainty. The variations of this factor gives the relative calibration accuracy. The standard deviation of the calibration factor is therefore a good estimator of the relative calibration accuracy. The absolute calibration will be determined finally on the whole sky map (see Sect. 3.4 for a first attempt). A mask on a region with IRAS $100 \mu \mathrm{m}$ emission above a given value can be applied in order to decrease the contribution from dust emission.

\subsection{Relative calibration accuracy}

\subsubsection{Effects of CMB anisotropies}

In this analysis, we remove regions with IRAS $100 \mu \mathrm{m}$ emission higher than $10 \mathrm{MJy} / \mathrm{sr}$ which is optimal, as will be shown in Sect. 3.3.3. Figure 4 shows the variations of the calibration factor for a HFI $100 \mathrm{GHz}$ channel along the simulated mission assuming known OM and PM dipoles characteristics, with and without CMB cosmological anisotropies. While the PM dipole varies along the mission, the OM dipole produces almost the same signal on each ring, as is apparent in Fig. 3. The accuracy with which the dipoles can be measured remains high even when the PM dipole signal on the scan is minimum, on rings around numbers 40 and 80 . When CMB cosmological anisotropies are not included, the relative variations of the calibration factor are very low, mainly dominated by noise effects. CMB anisotropies therefore dominate the uncertainties in measuring the amplitude of the dipole on individual rings. The accuracy improves when averaging over a larger number of rings. This part of the error goes exactly to zero when the whole sky is used. The accuracy of dipole fitting on HFI rings is therefore highly limited by the CMB cosmological anisotropies. This fit is equivalent to find the $m=1$ mode of the ring power spectrum $\Gamma_{m}$. The relation from Delabrouille et al. (1998a) links this spectrum with the $C_{\ell}$ spectrum:

$\Gamma_{m}=\sum_{\ell=|m|}^{\infty} W_{\ell m} C_{\ell}$

where $W_{\ell m}$ are window functions related to the beam shape and to the ring radius. The dipole term given by $\Gamma_{1}$ clearly depends on all multipole $\ell \geq 1$ of the CMB cosmological anisotropies. This coupling between modes disappear on a full map, indicating that the final absolute calibration should be done on the full map of Planck data as illustrated in Sect. 3.4. Nevertheless, relative calibration between rings at $100 \mathrm{GHz}$ is expected to be possible at a level of the order of a few percent as discussed in Sect. 3.3.3.

At $545 \mathrm{GHz}$ (Fig. 5), the signal is dominated by galactic dust emission and the accuracy of the CMB dipole calibration is about the same whether CMB cosmological anisotropies are considered or not. The relative calibration between rings at $545 \mathrm{GHz}$ is possible at a level of about $15 \%$.

\subsubsection{Shifted direction of the CMB dipole}

The PM velocity vector direction is known with an accuracy of about 14 arcmin at $1 \sigma$ (Lineweaver et al. 1996). To test the effect of this uncertainty on ring relative calibration, we fit the simulated data with a PM dipole shifted by 30 arcmin in all directions (about $2 \sigma$ from COBE results). Figure 6 shows the results obtained close to the worst case. The accuracy on the relative calibration is not highly affected and the calibration factor standard deviation is increased by only $15 \%$. These results show that a relative calibration on rings is still possible in an iterative way: with a first relative response correction and with enough observing time, the dipole direction will be improved and therefore the relative calibration will be enhanced. 
Table 1. Required sensitivities of Planck-HFI per unpolarised detection chain (expected sensitivities are twice better). The last two lines give the sensitivities per channel for the full mission.

\begin{tabular}{ccccccc}
\hline \hline Frequency (GHz) & 857 & 545 & 353 & 217 & 143 & 100 \\
Resolution (arcmin) & 5 & 5 & 5 & 5 & 7.1 & 9.2 \\
Number of detector $_{\mathrm{NET}_{\mathrm{CMB}}\left(\mu \mathrm{K} \mathrm{Hz}^{-0.5}\right)}$ & 4 & 4 & 4 & 4 & 4 & 4 \\
NEI $\left(\mathrm{MJy} \mathrm{sr}^{-1} \mathrm{~Hz}^{-0.5}\right)$ & 182000 & 3995 & 553 & 182 & 123 & 99 \\
Thermo. temperature sensitivity per ring $(\mu \mathrm{K})$ & 199000 & 4370 & 605 & 200 & 113 & 80 \\
Intensity sensitivity per ring $\left(\mathrm{MJy} \mathrm{sr}^{-1}\right)$ & $269 \times 10^{-3}$ & $232 \times 10^{-3}$ & $165 \times 10^{-3}$ & $88 \times 10^{-3}$ & $47 \times 10^{-3}$ & $23 \times 10^{-3}$ \\
Thermo. temperature sensitivity full mission $(\mu \mathrm{K})$ & $294 \times 10^{-3}$ & $253 \times 10^{-3}$ & $180 \times 10^{-3}$ & $96 \times 10^{-3}$ & $43 \times 10^{-3}$ & $19 \times 10^{-3}$ \\
\hline Intensity sensitivity full mission $\left(\mathrm{MJy} \mathrm{sr}^{-1}\right)$ & $54 \times 10^{-3}$ & $46 \times 10^{-3}$ & $33 \times 10^{-3}$ & $18 \times 10^{-3}$ & $6.6 \times 10^{-3}$ & $2.6 \times 10^{-3}$ \\
\hline \hline
\end{tabular}
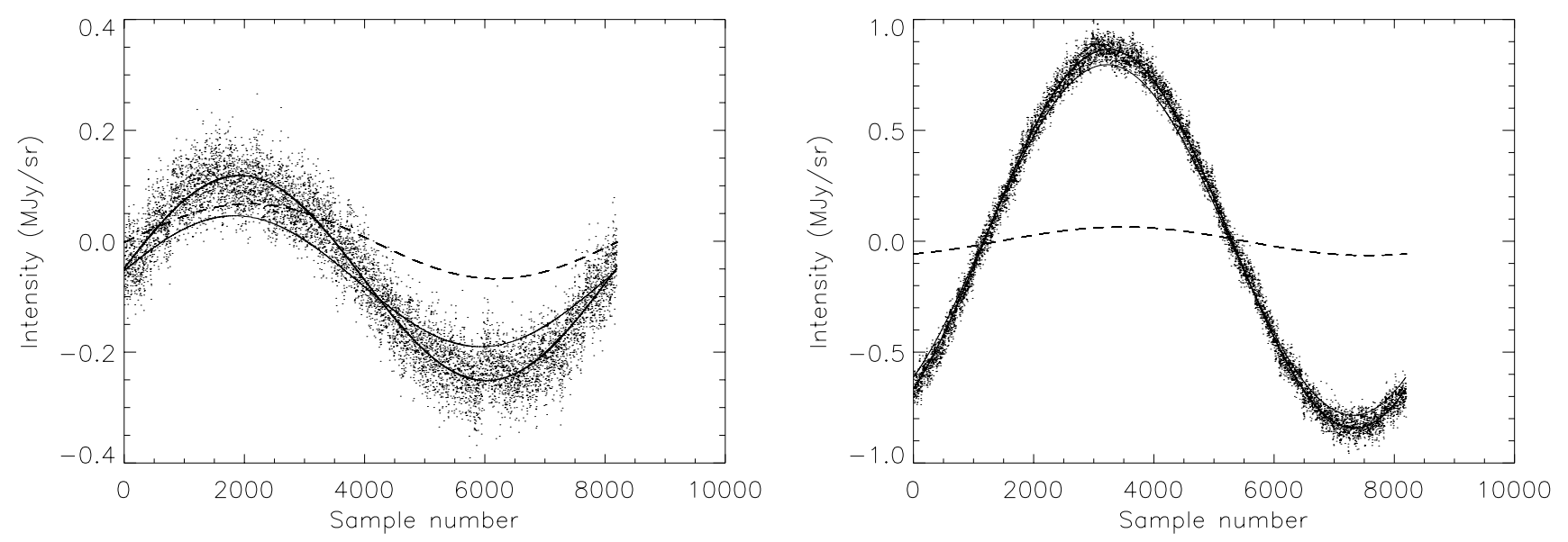

Fig. 3. Example of the analysis done at $100 \mathrm{GHz}$ on two rings (No. 0 and No. 20). The points represent the simulated signal. The PM and OM dipoles are represented by light solid and dashed curves respectively. The bold solid curve is the OM plus PM motion dipole fitted on the signal.

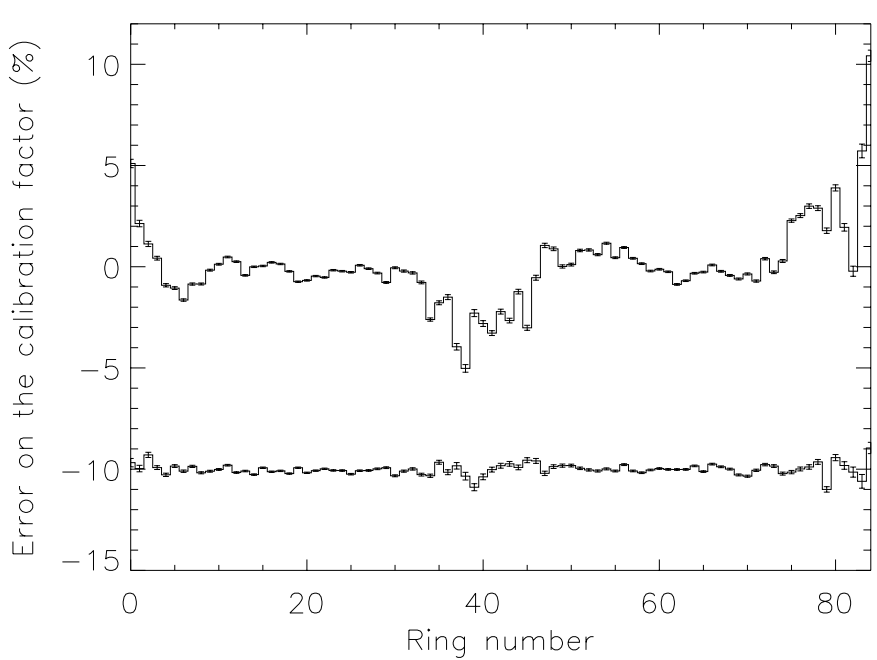

Fig. 4. Variations of the calibration factor for a HFI $100 \mathrm{GHz}$ channel as a function of the simulated ring number. We have assumed perfectly known OM and PM dipoles characteristics. The lower curve has been computed without CMB cosmological anisotropies and is shifted by $10 \%$ for clarity. Regions where IRAS $100 \mu \mathrm{m}$ emission greater than $10 \mathrm{MJy} / \mathrm{sr}$ were removed from the fit.

\subsubsection{Effect of dust contamination}

The effect of dust contamination on calibration can be tested by removing from the dipole fit the data points having an emission

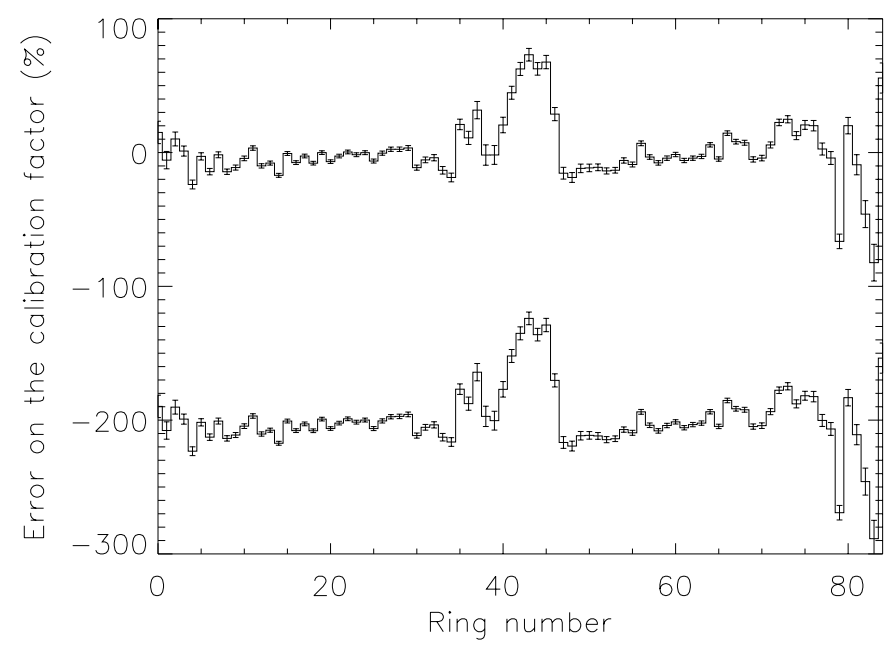

Fig. 5. Variations of the calibration factor for a HFI $545 \mathrm{GHz}$ channel as a function of the ring number. The legend is similar to Fig. 4. The lower curve is shifted by $200 \%$ for clarity.

in IRAS $100 \mu \mathrm{m}$ map above a given level. For very low cut-off values, the fit is degraded due to the decrease in the number of data points. On the other hand, with a high cut-off value, all the emission of dust in our Galaxy is taken into account in the fit and degrades the final accuracy. We therefore expect an optimum value to exist. In order to study this effect, the standard 


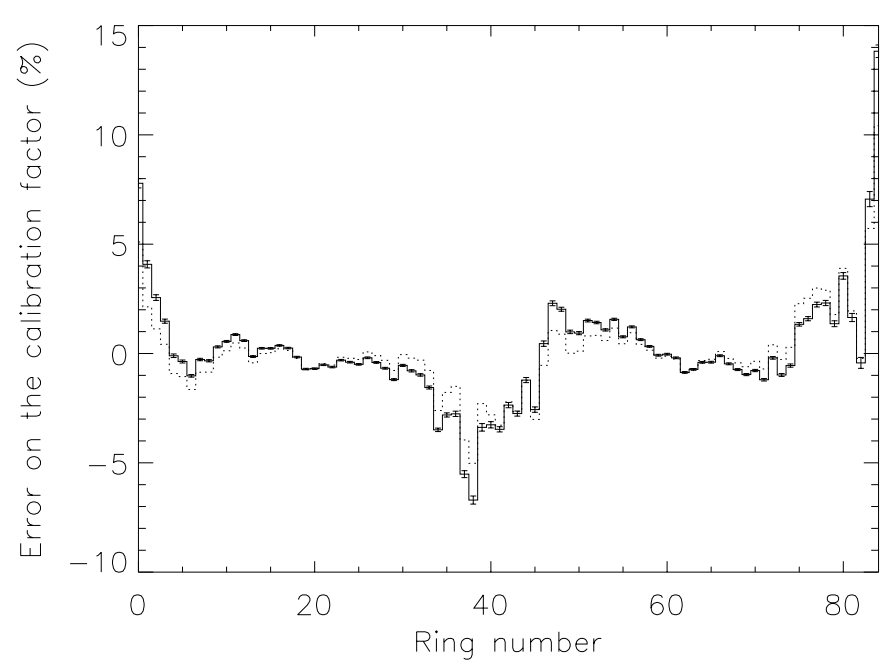

Fig. 6. Variations of the calibration factor for a HFI $100 \mathrm{GHz}$ channel as a function of the ring number with an error in the PM dipole direction of 30 arcmin. The dotted curve assumed a perfectly known PM dipole direction. Regions where IRAS $100 \mu \mathrm{m}$ emission is greater than $10 \mathrm{MJy} / \mathrm{sr}$ were removed from the fit.

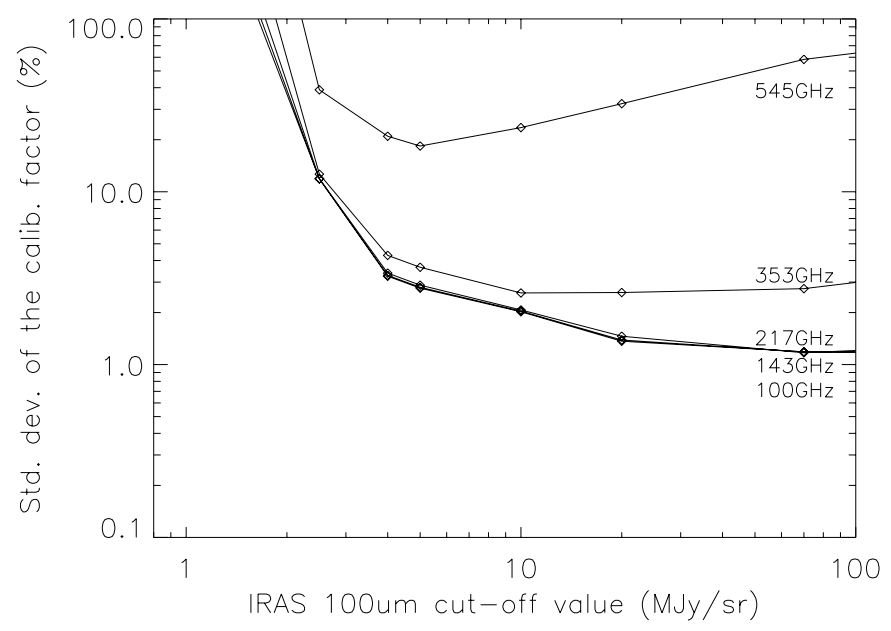

Fig. 7. Variations of the standard deviation of the calibration factor (expressed in percentage of the calibration factor) for all HFI channels, except the $857 \mathrm{GHz}$, as a function of the IRAS $100 \mu \mathrm{m}$ cut-off value.

deviation of the calibration factor on all rings is plotted in Fig. 7 as a function of the IRAS $100 \mu \mathrm{m}$ cut-off value.

On CMB channels (100 GHz, $143 \mathrm{GHz}$ and $217 \mathrm{GHz}$ ), increasing the IRAS $100 \mu \mathrm{m}$ cut-off value does not decrease the relative calibration accuracy: as shown in the previous section, this accuracy is limited only by CMB cosmological anisotropies. A relative calibration accuracy of $1.2 \%$ at $1 \sigma$ is possible on these channels. For higher frequency channels, the emission of galactic dust is more dominant and there is, as expected, an optimum cut-off value at a few MJy/sr. Near this optimum, an accuracy of about $15 \%$ in relative calibration can be expected at $545 \mathrm{GHz}$ using the dipole signal alone.

\subsection{Absolute calibration accuracy}

In order to test the accuracy of the absolute calibration on a global map, we have fitted simultaneously all simulated rings with a dipole term from OM and PM. This is an approximation of a full fit on the whole sky. Our procedure nevertheless gives a first approach to the global calibration which will be expensive in computer resources.

We can write the signal as:

$$
\begin{aligned}
\operatorname{Signal}(l, b)= & F \times\left[\operatorname{PM}_{\left(A_{0}, l_{0}, b_{0}\right)}(l, b)+\mathrm{OM}(l, b)\right. \\
& +\operatorname{Gal}(l, b)+\operatorname{CMBA}(l, b)]
\end{aligned}
$$

where $F$ is the calibration factor, $A_{0}$ is the PM dipole amplitude $\left(A_{0}=3.36 \mathrm{mK}\right),\left(l_{0}, b_{0}\right)$ is the PM dipole direction $\left(l_{0}=264.31^{\circ}, b_{0}=48.05^{\circ}\right), \mathrm{Gal}$ is the Galactic signal and CMBA the CMB anisotropy contribution. This analysis has been done only on CMB channels $(100 \mathrm{GHz}, 143 \mathrm{GHz}$ and $217 \mathrm{GHz}$ ). We remove CMB cosmological anisotropies from the fit since, in our case, they introduce an $\ell=1$ term which will not be present when using the whole sky. In order to remove the dust contamination effect, we fit only parts of the rings where IRAS $100 \mu \mathrm{m}$ emission is lower than $15 \mathrm{MJy} / \mathrm{sr}$. We assume in the procedure that we perfectly know only the $\mathrm{OM}$ dipole so that the calibration is due to this term. We first search for the best $F, A_{0}, l_{0}$ and $b_{0}$ and their accuracy by fitting simultaneously the 84 ring signals of one CMB channel. This analysis shows that we can separate our problem into two much faster fitting procedures: one to get the best calibration factor and PM dipole amplitude, and the other to find the best PM dipole direction. These two problems are mostly decorrelated since a change in the PM dipole direction cannot be compensated by a change in its amplitude (or in the calibration factor). This lead to $\chi^{2}$ contour plots shown in Figs. 8 and 9. We obtain the following results for one $100 \mathrm{GHz}$ detection chain:

- we can recover the PM dipole direction at better than 1.7 arcmin (Fig. 8);

- we find $A_{0}=(3.374 \pm 0.007) \mathrm{mK}$ at a $95 \%$ confidence level and

- $F$ is found to be equal to $0.996 \pm 0.002$ at a $95 \%$ confidence level (Fig. 9).

This shows on the one hand that Planck-HFI will be able to make a new and accurate determination of the PM dipole direction. On the other hand, by combining the error on $F$ and $A_{0}$ and considering the $\mathrm{OM}$ dipole as an absolute calibrator, we see that we can reach an absolute calibration level better than $0.4 \%$ for HFI at low frequency. This accuracy is very good even if quite pessimistic since it takes into account only part of Planck-HFI one year data.

\section{Detectability of the dipole effect of the Far InfraRed Background (FIRB)}

The FIRB is due to the integrated emission of distant redshifted galaxies. Its SED between $100 \mu \mathrm{m}$ and $1 \mathrm{~mm}$ is well represented by the following expression (Lagache et al. 1999):

$I(v)=8.8 \times 10^{-5}\left(\frac{v}{v_{0}}\right)^{1.4} P_{v}\left(T_{0}\right)$ 


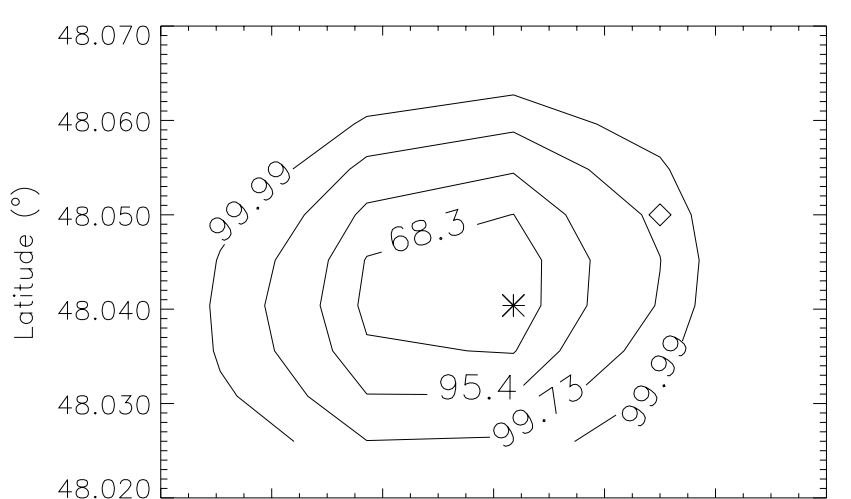

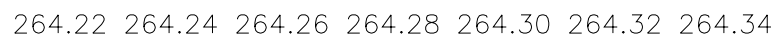
Longitude $\left({ }^{\circ}\right)$

Fig. 8. Contour plot in the $l / b$ plane of the $\chi^{2}$ obtained for the fit of the PM dipole direction, expressed in percentage of confidence level, for a $100 \mathrm{GHz}$ channel. We assumed that the PM dipole amplitude and instrument response for this fit are known (see text). The asterisk represents the best fit while the diamond is the input dipole direction. Regions with IRAS $100 \mu$ m emission higher than $15 \mathrm{MJy} / \mathrm{sr}$ were removed from the fit.

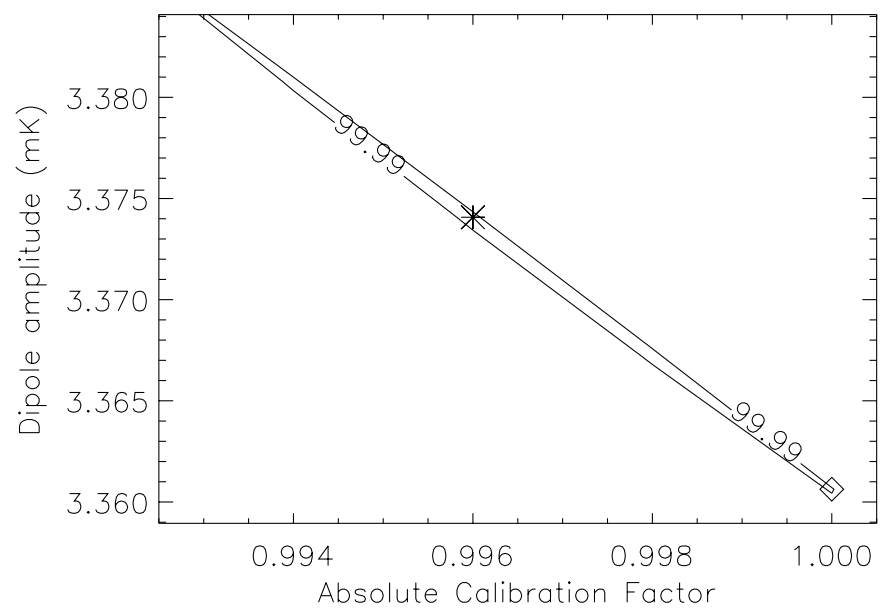

Fig. 9. Contour plot in the $F / A_{0}$ plan of the $\chi^{2}$ obtained for the fit of the PM dipole amplitude and instrument response, expressed in percentage of confidence level, for a $100 \mathrm{GHz}$ channel. We assumed that the PM dipole amplitude and instrument response for this fit are known (see text). The asterisk point represents the best fit while the diamond is the input of the simulation. Regions with IRAS $100 \mu \mathrm{m}$ emission higher than $15 \mathrm{MJy} / \mathrm{sr}$ were removed from the fit.

where $v_{0}=100 \mathrm{~cm}^{-1}, T_{0}=13.6 \mathrm{~K}$ and $P_{v}(T)$ is the Planck function. This expression has been obtained by averaging over multiple clean regions, almost homogeneously distributed on the sky, where dust contamination is low. The FIRB dipole spectrum can be deduced from its monopole spectrum by using Eqs. (2) and (6):

$I_{\text {dip FIRB }}(v)=\beta I(v)\left(\frac{x \mathrm{e}^{x}}{\mathrm{e}^{x}-1}-1.4\right)$

where $x=h v /\left(k T_{0}\right)$.

In this section, we analyse the detection of the FIRB dipole by Planck-HFI which, if detected, could give indirect information on the monopole term not accessible with this instrument.

\subsection{Signal to noise ratio}

\subsubsection{White noise case}

In order to evaluate the expected sensitivity of HFI on the FIRB dipole signal, we first consider the case of instrumental noise having a pure white spectrum. As for the $\mathrm{CMB}$ dipole, the FIRB dipole will be best detected on a full sky map where no aliasing from CMB anisotropies will occur. An estimate of the Signal-to-Noise Ratio (SNR) can be done on the $\ell=1$ multipole of the angular power spectrum decomposition. The expected $C_{1}$ for a dipole signal having an amplitude $A$ is given by:

$C_{1}=\frac{4 \pi}{9} A^{2}$

Knox (1995) and Tegmark (1997) have shown that the effect of uniform instrumental noise can be accurately modeled as an additional random field on the sky, with an angular power spectrum given by:

$C_{\ell \text { noise }}=\Omega_{\mathrm{b}} \sigma^{2}$

where $\Omega_{\mathrm{b}}$ is the beam solid angle and $\sigma$ is the rms noise per pixel. The SNR on $C_{1}$ is therefore equal to $S N R=C_{1} / C_{1}$ noise and the results are summarised in Table 2. It shows that the FIRB dipole can be detected at $857 \mathrm{GHz}$ and $545 \mathrm{GHz}$, but not at $353 \mathrm{GHz}$, with averaging over at least $10^{\circ}$ if the detection noise is white.

\subsubsection{Effect of the $1 / f$ noise}

The HFI will exhibit $1 / f$ noise mainly due to the readout electronics (Gaertner et al. 1997; Piat et al. 1997), temperature fluctuations of the cryogenic system (Piat et al. 1999) and from far side lobe signal (Delabrouille 1998b). The assumed knee frequency is $10 \mathrm{mHz}$ for HFI in order to have enough stability on a timescale of $1 \mathrm{~min}$ corresponding to the spin period of the satellite (Piat et al. 1999). A destriping algorithm can remove an important fraction of low frequency drifts. Delabrouille (1998c) has shown that removing fluctuations on timescales larger than the spin period of Planck is possible thanks to redundancies. A conservative analysis could be made by assuming that all slow components of the noise, except frequencies lower than the spin frequency, are not filtered. Equation (3) allows us to express the ring anisotropy power spectrum $\Gamma_{m}$ with the $C_{\ell}$ angular power spectrum on the sky. The window functions $W_{\ell m}$ are given by Delabrouille et al. (1998a):

$W_{\ell m}=B_{\ell} \mathcal{P}_{\ell m}^{2}(\Theta)$

where $\Theta$ is the ring angular radius, $B_{\ell}$ is the beam response function (assuming a symmetric beam) and $\mathcal{P}_{\ell m}$ are given by the following expression where $P_{\ell m}$ are the associated Legendre polynomials: $\begin{aligned} \mathcal{P}_{\ell m}(\theta) & =\sqrt{\left(\frac{2 \ell+1}{4 \pi}\right) \frac{(\ell-m) !}{(\ell+m) !}} P_{\ell m}(\cos \theta), \quad \text { for } m \geq 0, \\ & =(-1)^{|m|} \mathcal{P}_{\ell|m|}(\theta), \quad \text { for } m<0\end{aligned}$ 
Table 2. FIRB dipole level in the Planck-HFI channels and SNR for three pixelisations assuming a white detector noise only. We also assumed a 1.17 year mission, square pixels with size given by $F W H M$ and bolometer noise given in Table 1.

\begin{tabular}{cccc}
\hline \hline Frequency $(\mathrm{GHz})$ & 857 & 545 & 353 \\
FIRB dipole intensity amplitude (MJy/sr) & $1.6 \times 10^{-3}$ & $3.4 \times 10^{-4}$ & $4.9 \times 10^{-5}$ \\
SNR per pixel & 0.04 & 0.01 & 0.00 \\
SNR per pixel of $1^{\circ}$ & 0.50 & 0.12 & 0.02 \\
SNR per pixel of $10^{\circ}$ & 5.0 & 1.2 & 0.2 \\
\hline \hline$C_{1 \text { FIRB }}\left(\left(\mathrm{MJy} / \mathrm{sr}^{2} \mathrm{rad}^{2}\right)\right.$ & $3.5 \times 10^{-6}$ & $1.7 \times 10^{-7}$ & \multirow{2}{*}{$3.4 \times 10^{-9}$} \\
$C_{1 \text { noise }}\left((\mathrm{MJy} / \mathrm{sr})^{2} \mathrm{rad}^{2}\right)$ & $6.1 \times 10^{-9}$ & $4.6 \times 10^{-9}$ & $2.3 \times 10^{-9}$ \\
SNR full map on $C_{1}$ & 565 & 36 & 1.5 \\
\hline
\end{tabular}

Equation (3) can be used to deduce the angular power spectrum of the instrumental noise from the Noise Equivalent Power (NEP) spectrum.

A mode $m$ on a ring has an equivalent frequency bandwidth of $1 /\left(2 T_{\text {spin }}\right)$ where $T_{\text {spin }}$ is the spin period. Furthermore, the noise power spectrum has to be filtered by the beam filter in order to obtain the noise spectrum projected on the sky. The NEP is therefore related to the ring power spectrum of the noise by:

$N E P^{2}(f) B^{2}(f)=2 T_{\text {spin }} \Gamma_{m}$

where $f=m / T_{\text {spin }}$ is the frequency. $B(f)$ is the beam filter given by the following expression in the case of a Gaussian beam shape of Full Width Half Maximum $F W H M=$ $\sigma_{b} \sqrt{8 \ln 2}$ :

$B(f)=\exp \left[-\frac{1}{2}\left(f \frac{\sigma_{b} T_{\text {spin }}}{\sin \Theta}\right)^{2}\right]$.

Equation (3) can therefore be expressed in terms of NEP:

$N E P^{2}\left(f=m / T_{\text {spin }}\right)=\frac{2 T_{\text {spin }}}{B^{2}(f)} \sum_{\ell=|m|}^{\ell_{\text {max }}} C_{\ell \text { noise }} W_{\ell m}$

where the sum is carried over all accessible $\ell$. The value of $\ell_{\max }$ is given by the highest frequency $f_{\max }$ transmitted by the readout electronics, about $100 \mathrm{~Hz}$ for $\mathrm{HFI}$, that leads to $\ell_{\max }=$ $T_{\text {spin }} f_{\max } / \sin \Theta=6000$ for a ring angular radius $\Theta=90^{\circ}$.

Equation (13) can be viewed as a matrix multiplication:

$N E P^{2}=M \times C_{\text {noise }}$

where $N E P$ and $C_{\text {noise }}$ are vectors containing $N E P(f=$ $\left.m / T_{\text {spin }}\right)$ and $C_{\ell \text { noise }}$ respectively. $\mathrm{M}$ is a triangular matrix since $\Gamma_{m}$ depends only on $C_{\ell}$ with $\ell \geq|m|$, and the solution is therefore quite easy to obtain. With this method, we get $C_{\ell \text { noise }}$ assuming a noise spectrum of Planck-HFI given by:

$N E P^{2}(f)=N E P_{0}^{2}\left[1+\left(\frac{f_{\text {knee }}}{f}\right)^{\alpha}\right]$

where $N E P_{0}$ is the $N E P$ value in the white part of the noise, $f_{\text {knee }}$ is the knee frequency and $\alpha$ a constant. The effect of $1 / f$ noise on the angular power spectrum is shown in Fig. 10 for a knee frequency of $10 \mathrm{mHz}$ in 4 different cases of noise behaviour. The SNR on $C_{1}$ will be degraded by a maximum factor of about 270 which nevertheless allows a detection at more than $2 \sigma$ at $857 \mathrm{GHz}$.

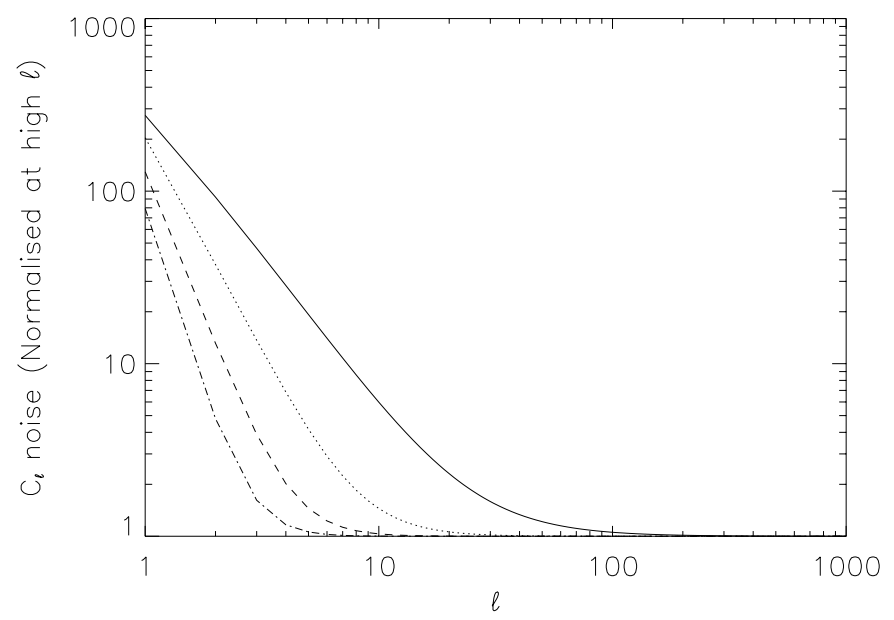

Fig. 10. Effect of $1 / f$ noise on the noise angular power spectrum $C_{\ell \text { noise }}$ normalised at high $\ell$, for a knee frequency of $10 \mathrm{mHz}$. The solid curve correspond to a power $\alpha=1$ (see Eq. (15)) while the dotted, dashed and dot-dashed lines are for $\alpha=2,3$ and 4 respectively.

\subsection{Estimation of the total dipole spectrum}

In order to evaluate the effect of calibration and component separation on the detectability of the FIRB dipole, the relative level of FIRB dipole with respect to other components has to be evaluated. The total dipole signal in Planck-HFI channels is produced by the $\mathrm{CMB}$, the FIRB, the inhomogeneous dust distribution in the Galaxy and the dipole term in the IPD emission. The analysis described in Sect. 3.1 showed that this later contribution at highest frequencies is about an order of magnitude lower than the FIRB dipole term. The IPD emission is therefore assumed to be negligible.

The repartition of dust in the Galaxy produces a dipole signal, the so-called "dust dipole", that is not due to the Doppler effect. This signal has been estimated on the COBE-DIRBE sky map at $100 \mu \mathrm{m}, 140 \mu \mathrm{m}$ and $240 \mu \mathrm{m}$. In order to remove strong emission from the galactic plane, a cut in galactic latitude or a cut-off in maximum emission level has been applied. Both methods lead to about the same results for intermediate cut, within less than $20^{\circ}$ on the fitted dipole direction and about $10 \%$ accuracy on its amplitude. The dipole that dust mimic is pushed toward the galactic south pole as the cut in the latitude is increased, or the cut-off in maximum emission is decreased. A reasonable cut in galactic latitude of $\pm 15^{\circ}$ has therefore been applied. These data have been used to interpolate the 


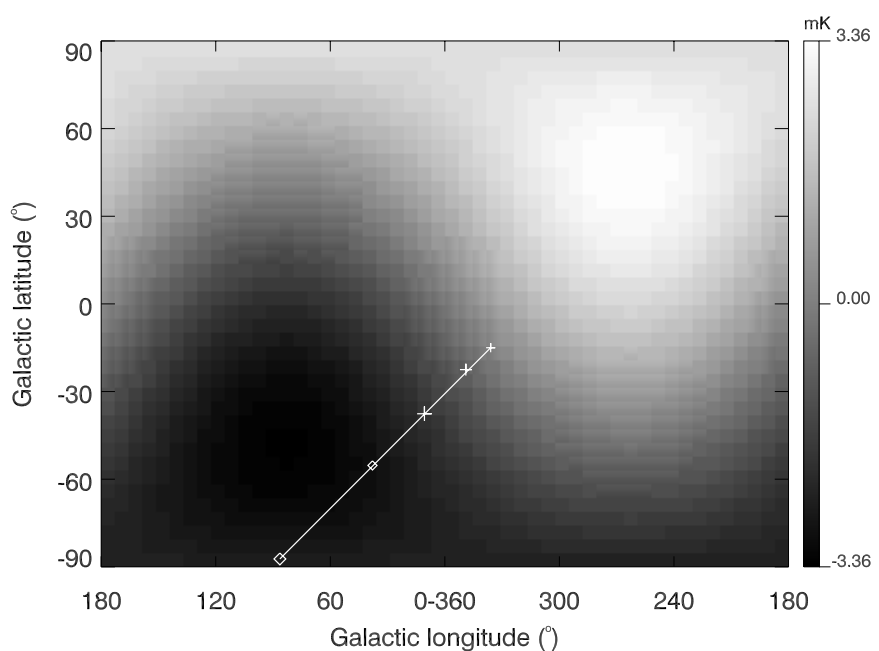

Fig. 11. Dust dipole directions superimposed on the CMB dipole. The crosses are data points obtained with COBE-DIRBE data at $100 \mu \mathrm{m}$, $140 \mu \mathrm{m}$ and $240 \mu \mathrm{m}$ with increasing size of the symbol, assuming a cut in galactic latitude of $\pm 15^{\circ}$. These points have been extrapolated to HFI $350 \mu \mathrm{m}(857 \mathrm{GHz})$ and $550 \mu \mathrm{m}(545 \mathrm{GHz})$ channels represented by diamonds with increasing size.

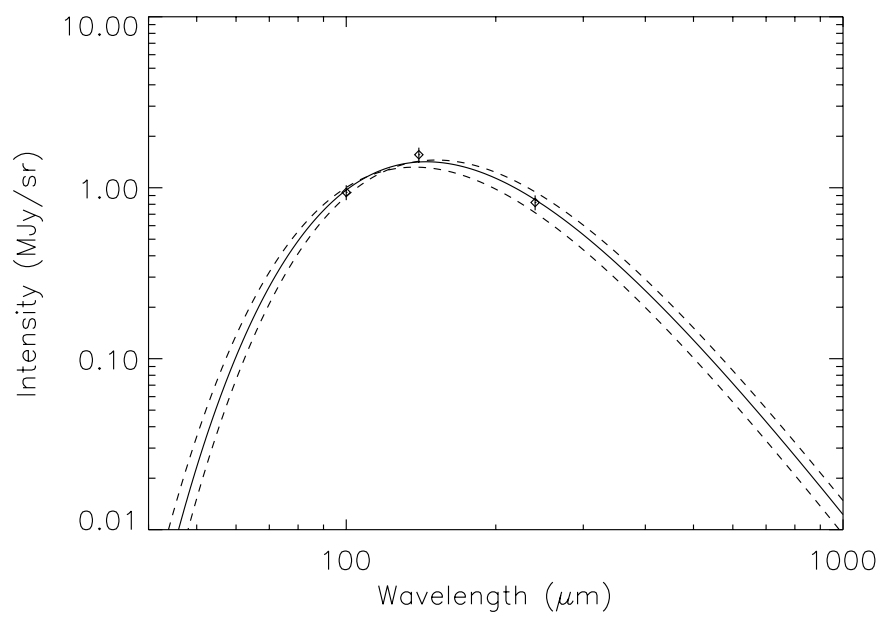

Fig. 12. Spectrum of the dust dipole. The diamonds are the points obtained with COBE-DIRBE assuming a cut in galactic latitude of $\pm 15^{\circ}$. The solid line is the best fit with a $\lambda^{-2}$ emissivity leading to a dust temperature of $(20 \pm 1) \mathrm{K}$ and a $\mathrm{H} \mathrm{I}$ column density of $(5.2 \pm 1.5) 10^{19} \mathrm{~cm}^{-2}$. The dashed lines gives the upper and lower limits.

dust dipole on Planck-HFI $545 \mathrm{GHz}$ and $857 \mathrm{GHz}$ as shown in Figs. 11 and 12. The dipole direction has been extrapolated linearly with galactic coordinate, assuming a $\pm 20^{\circ}$ uncertainty. It seems to go in the direction of the south galactic pole with increasing wavelength, meaning that only the north/south asymmetry will be seen. The dust dipole spectrum follows a $(20 \pm 1) \mathrm{K}$ blackbody modified by a $v^{2}$ emissivity and with a H I column density of $(5.2 \pm 1.5) 10^{19} \mathrm{~cm}^{-2}$. We also assumed that $90 \%$ of this signal can be removed by component separation. The resulting dust dipole spectrum with its uncertainty, projected on the CMB dipole axis, is shown in Fig. 13 together with the CMB, FIRB and total dipole. The FIRB dipole signal is only about $10 \%$ of the total dipole which is dominated

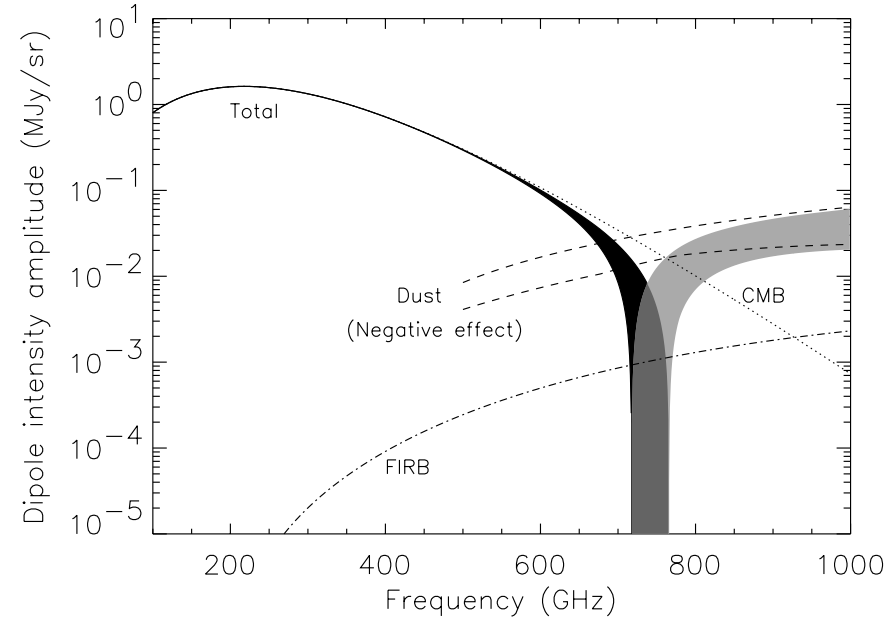

Fig. 13. SED of dipoles from the CMB (dotted line), dust assuming that $90 \%$ is removed (dipole projected on the CMB dipole direction, dashed lines giving lower and upper limits) and the FIRB (dot-dashed line). The solid region gives the spectrum of the total dipole with the uncertainty on the dust dipole, the black one of the positive dipole while the grey one is for the negative dipole.

by the dust contribution for frequencies higher than $700 \mathrm{GHz}$. This has two consequences:

1. To recover the FIRB dipole signal, the dust dipole has to be removed using component separation methods at the $1 \%$ accuracy level (10 times better than currently assumed) which will be difficult to achieve, even on large scales;

2. the calibration of the $545 \mathrm{GHz}$ and $857 \mathrm{GHz}$ channels will be obtained by comparison with the galactic plane emission measured with COBE-FIRAS. The accuracy in these channels will probably be limited by the COBE-FIRAS calibration accuracy which is about $3 \%$, a factor of 3 higher than needed to detect the FIRB dipole effect.

The detection by Planck-HFI of the FIRB dipole is therefore unfortunately very difficult, due to the component separation and calibration accuracies of the high frequency channels.

\section{Conclusion}

We have shown that we can use the PM and OM dipoles observation for relative and absolute calibration for Planck-HFI. For CMB channels, we reach a relative calibration accuracy of about $1.2 \%$ on rings. An absolute calibration accuracy better than $0.4 \%$ has been obtained when the whole sky is used. At the end, the Planck-HFI absolute calibration will be limited by the uncertainty on the CMB temperature. Moreover, we show that the CMB dipole direction will be improved by Planck. The next step consists of a detailed study of systematic effects on calibration.

The detectability of the FIRB dipole has also been studied and we have shown that such study is a difficult challenge due mainly to dust contamination. 


\section{References}

Bernstein, R. A., Freedman, W. L., \& Madore, B. F. 2001 [astro-ph/0112153]

Blake, C., \& Wall, J. 2002, Nature, 416, 150

Boulanger, F., Abergel, A., Bernard, J. P., et al. 1996, A\&A, 312, 256

Cambrésy, L., Reach, W. T., Beichman, C. A., \& Jarrett, T. H. 2000, ApJ, 555, 563

Delabrouille, J., Górski, K. M., \& Hivon, E. 1998, MNRAS, 298, 445

Delabrouille, J. 1998, Ph.D. Thesis

Delabrouille, J. 1998, A\&AS, 127, 555

Dwek, E., Arendt, R. G., Hauser, M. G., Fixsen, D., et al. 1998, ApJ, 508, 106

Fixsen, D. J., Cheng, E. S., Gales, J. M., et al. 1996, ApJ, 473, 576

Fixsen, D. J., Dwek, E., Mather, J. C., et al. 1998, ApJ, 508, 123

Gaertner, S., Benoit, A., Lamarre, J. M., et al. 1997, A\&AS, 126, 151

Giard, M., Hivon, E., Nguyen, C., et al. 1999 [astro-ph/9907208]

Gispert, R., Lagache, G., \& Puget, J. L. 2000, A\&A, 360, 1

Gorjian, V., Wright, E. L., \& Chary, R. R. 2000, ApJ, 536, 550

Górski, K. M., Hivon, E., \& Wandelt, B. D. 1998, in Proc. of the MPA/ESO Cosmology Conf., Evolution of Large Scale Structure, ed. A. J. Banday, R. S. Sheth, \& L. Da Costa

Halpern, M., \& Scott, D. [astro-ph/9904188]

Hauser, M. G., Arendt, R. G., Kelsall, T., et al. 1998, ApJ, 508, 25

Janssen, M., Scott, D., White, M., et al., 1996 [astro-ph/9602009]

Knox, L. 1995, Phy. Rev. D, 52(8), 4307
Kelsall, T., et al. 1998, ApJ, 508, 44

Kogut, A., Lineweaver, C., Smoot, G. F., et al. 1993, ApJ, 419, 1

Lagache, G., Abergel, A., Boulanger, F., et al. 1999, A\&A, 344, 322

Lagache, G., Haffner, L. M., Reynolds, R. J., \& Tufte, S. L. 2000, A\&A, 354, 247

Lamarre, J. M. 1998, Proc. of the workshop on The Cosmic Microwave Background and the Planck mission, 22-26 June 1998, Santander, Spain

Lamarre, J. M. 2001, HFI Instrument Working Group, 27-28 September 2001 held in IAS, Orsay, France

Lauer, T. R., \& Postman, M. 1994, ApJ, 425, 418

Lineweaver, C. H., Tenorio, L., Smoot, G. F., et al. 1996, ApJ, 470, 38

Piat, M., Lamarre, J. M., Benoit, A., et al. 1997, Proc. 7th Int. Workshop on Low Temperature Detectors LTD-7, 27 July-2 August 1997, Munich, Germany, pub. by MPI Physik, ISBN 3-00-002266-X, avail. from urg@mppmu.mpg. de

Piat, M., Leriche, B., Torre, J. P., et al. 1999, NIMA 444, 413-418

Pozzetti, L., Madau, P., Zamorani, G., et al. 1998, MNRAS, 298, 1133

Puget, J. L., Abergel, A., Bernard, J. P., et al. 1996, A\&A, 308, L5

Renault, C., Barrau, A., Lagache, G., \& Puget, J.-L. 2001, A\&A, 371, 771

Scaramella, R., Vettolani, G., \& Zamorani, G. 1991, ApJ, 376, L1

Seljak, U., \& Zaldarriaga, M., 1996, ApJ, 469, 437

Stanev, T., \& Franceschini, A. 1998, ApJ, 494, L159

Tegmark, M. 1997, Phy. Rev. D, 56(8), 4514

Wright, E. L. 2001, ApJ, in press 